\title{
Unravelling how Climate and Competition Shape Sockeye Salmon Dynamics Across the Northeast Pacific Ocean
}

\author{
Brendan Connors ${ }^{1}$, Michael Malick ${ }^{2}$, Gregory T. Ruggerone ${ }^{3}$, Pete Rand ${ }^{4}$, Milo Adkison ${ }^{5}$, James R. Irvine, \\ Rob Campbell ${ }^{4}$, and Kristen Gorman ${ }^{4}$ \\ ${ }^{1}$ Fisheries and Oceans Canada, Institute of Ocean Sciences, 9860 W. Saanich Rd, Sidney, BC V8L 5T5 Canada \\ ${ }^{2}$ Oregon State University, Cooperative Institute for Marine Resources Studies, 2030 SE Marine Science Drive, \\ Newport, OR 97365, USA \\ ${ }^{3}$ Natural Resources Consultants, Inc., Seattle, WA 98028, USA \\ ${ }^{4}$ Prince William Sound Science Center, PO Box 705, 300 Breakwater Avenue, Cordova, Alaska, 99574 USA \\ ${ }^{5}$ University of Alaska Fairbanks, College of Fisheries and Ocean Sciences, Juneau, AK 99801, USA \\ ${ }^{6}$ Fisheries and Oceans Canada, Pacific Biological Station, 3190 Hammond Bay Road, Nanaimo BC V9T 6N7 \\ Canada
}

Keywords: sockeye salmon, competition, ocean warming, survival, life history diversity

It is well known that both ocean conditions and inter- and intra-specific interactions can influence salmon growth and survival. However, to date, there has been little analysis of the potential mediating effects of ocean conditions on density-dependent interactions among salmon at sea. Such mediating effects may occur, for example, as a result of climate-induced reductions in growth during early marine life leading to increased sensitivity to density-dependent effects later in marine life. Alternatively, favorable ocean conditions during early marine life may mask the detection of density-dependent interactions or mediate their potential effects. Life-history diversity (e.g., variable age at ocean entry), may also influence the effects of ocean conditions and competition at sea on sockeye survival by spreading the consequences of negative climate or competitive interactions across multiple life histories within a given brood year. However, how and when life-history diversity mediates the influence of the conditions salmon experience at sea has also received little attention to date.

In this study, we set out to quantify how a warming ocean, and increasing competition with other salmon, interact to determine the survival of sockeye across their range, and how life history diversity mediates the effects of density-dependent interactions among salmon at sea. We compiled data from 46 sockeye salmon populations across the eastern North Pacific Ocean, spanning a broad geographic range of ocean entry points and life history diversity. We also compiled information on indices of ocean conditions during early marine life (SST anomalies within 400 $\mathrm{km}$ of each population's ocean entry point) and potential salmon competitors (total north Pacific pink salmon abundance). We then fit Bayesian hierarchical models to these data to estimate both the stock specific and regionwide effects of ocean conditions, competition and an interaction between the two on sockeye salmon productivity. We found that the productivity of sockeye from the southern portion of their range was inversely correlated with both ocean conditions and the abundance of competitors and that the effects of competition were stronger when the ocean was warmer. Conversely, for sockeye from the northern portion of their range, we found that productivity was positively correlated with a warming ocean and that there was limited evidence for a negative effect of competition.

We also examined empirical data to evaluate whether variation in smolt-age mediated the effects of competition. We found that that the estimated effect of competition on individual Sockeye salmon populations tended to become weaker (i.e., less negative) as the degree of variation in age at ocean entry increased.

Our findings provide a macroecological foundation upon which to consider how interactions among a changing ocean, inter-specific competition, and the erosion of life history diversity may shape the dynamics of sockeye salmon populations across the eastern North Pacific. 